\title{
Digging deeper into epigenetic regulation
}

Why do some individuals become addicts or suffer from psychiatric disorders such as depression whereas others escape these conditions despite exposure to drugs or stress? Recently it has become clear that epigenetic mechanisms might provide an answer to this question. A new study takes us a step closer to understanding how this might happen, revealing that histone deacetylase 5 (HDAC5) is a key mediator of the effects of chronic stress and drug exposure on the brain.

HDAC5 represses gene expression by altering chromatin structure. In their study, Nestler and colleagues showed that, in mice that were chronically exposed to cocaine, a single dose of the drug elevated HDAC5 phosphorylation in the nucleus accumbens (NAc), a region that is central to the brain's reward system. This phosphorylation leads to the translocation of the enzyme out of the nucleus, effectively abolishing its effects on gene expression.
To examine the functional effects of HDAC5 activity, the authors used viral-mediated gene transfer to overexpress the enzyme in the NAc while the mice were trained to associate a particular area of a testing chamber with receiving cocaine. Increased HDAC5 levels reduced the rewarding effect of the drug, as the mice were less likely to return to the same location when given a free choice. Furthermore, the authors showed that transgenic mice that lacked HDAC5 could be more effectively sensitized to the rewarding effects of cocaine by prior exposure to the drug than normal mice. This suggests that variations in the activity of HDAC5 between individuals might result in different responses to chronic rewarding stimuli.

To determine which genes are affected by changes in HDAC 5 activity, the authors carried out a genomewide microarray. This revealed alterations in the expression of 172 genes in the NAc of Hdac5-knockout mice that had been chronically exposed to cocaine, many of which had previously been linked to reward pathways.

Abnormalities in the response to chronic stress might contribute to depression. The authors therefore investigated the possibility that HDAC5 might mediate these responses. Social defeat, a chronicstress paradigm, downregulated HDAC5 mRNA levels in the NAc, whereas antidepressant treatment increased HDAC5 expression. Furthermore, Hdac5-knockout mice were more severely affected by chronic stress, showing signs of a depressive-like condition.

This study suggests that HDAC5 is regulated in response to chronic stressful or rewarding stimuli, and that its activity is central to the behavioural adaptations that occur in response to such exposure. It would be interesting to determine whether alterations in HDAC5 activity are predictive of susceptibility to psychiatric disorders in humans. Future work in this area might lead to a better understanding and improved treatment of these conditions.

Katherine Whalley

ORIGINAL RESEARCH PAPER Renthal, W. et al. Histone deacetylase 5 epigenetically controls behavioural adaptations to chronic emotional stimuli. Neuron 56, 517-529 (2007) FURTHER READING Tsankova, R., Renthal, W., Kumar, A. \& Nestler, E. J. Epigenetic regulation in psychiatric disorders. Nature Rev. Neurosci. 8 , 355-367 (2007) 